\title{
ON DECOUPLED QUANTIFICATION OF ENERGY DISSIPATION MECHANISMS IN TOROIDAL RING GYROSCOPES
}

\author{
Yusheng Wang ${ }^{1 *}$, Yu-Wei Lin ${ }^{1}$, Janna Rodriguez ${ }^{2}$, Gabrielle D. Vukasin ${ }^{2}$, Dongsuk D. Shin ${ }^{2}$, Hyun-Keun Kwon ${ }^{2}$, \\ David B. Heinz', Yunhan Chen ${ }^{2}$, Dustin D. Gerrard', Thomas W. Kenny', and Andrei M. Shkel ${ }^{1}$ \\ ${ }^{1}$ MicroSystems Laboratory, University of California, Irvine, CA, USA \\ ${ }^{2}$ Micro Structures \& Sensors Lab, Stanford University, CA, USA
}

\begin{abstract}
We present a study on the quantification of the energy dissipation mechanisms in Toroidal Ring Gyroscopes (TRG). Our approach is based on suppression of the viscous air damping and controlling thermoelastic dissipation (TED), thus exposing the anchor loss of the device. During testing, energy dissipative factors, such as viscous air damping, TED, and surface loss, were minimized by venting the encapsulation, pumping down to ultrahigh vacuum (UHV), and sweeping the temperature down to $123 \mathrm{~K}$, so that anchor loss became the dominant factor that influenced the overall quality factor of the device. The anchor loss limit of the tested TRG was measured to be 1.35 million, and this value was on the same level as predicted by PML method. This study demonstrates the use of a methodology for analysis of energy dissipation mechanisms in MEMS devices and the possibility of quantifying the contribution of each mechanism independently.
\end{abstract}

\section{INTRODUCTION}

Quality factor is a parameter describing the energy loss of vibratory MEMS devices [1]. It is used as a metric to optimize device design in order to enhance its performance. In Coriolis Vibratory Gyroscopes (CVG), for example, lower energy consumption and better in-run noise performance, such as bias stability and Angle Random Walk (ARW), can be achieved with a higher quality factor. The quality factor of a resonator is limited by many energy dissipation mechanisms, such as viscous air damping, TED, anchor loss, surface loss, and intrinsic material loss. The total quality factor of a vibratory structure can be expressed as

$$
Q_{\text {total }}^{-1}=Q_{\text {viscous }}^{-1}+Q_{T E D}^{-1}+Q_{\text {anchor }}^{-1}+Q_{\text {surface }}^{-1}+Q_{\text {others }}^{-1} .
$$

Viscous air damping increases rapidly as the resonator's surface-to-volume ratio increases [2]. Therefore, its effect is more dominant in micro-fabricated resonators than in devices of larger scales. Viscous air damping is proportional to air pressure in the molecular region, which for a device sealed with an ideal gas scales as the inverse square root of temperature. Viscous air damping can be suppressed by packaging and vacuum sealing. TED is related to the coupling of thermal and elastic deformation fields of the resonator through the Coefficient of Thermal Expansion (CTE) of the material $[3,4]$. TED can be reduced by optimizing the design [5], or building devices with materials with lower CTE, such as Fused Quartz [6]. Anchor loss is due to vibratory wave propagation from the resonator into the substrate through the anchor and it is related to the mode shape, mechanical properties of the substrate, and anchor geometry. A numerical technique called Perfectly Matched Layer (PML) is available in the COMSOL MultiPhysics Package, and has become popular for modeling anchor loss in resonating MEMS [1]. Dynamic balancing of the resonator [7] and careful anchor designs [8] have been demonstrated to reduce the anchor loss in resonators. The surface loss is related to the dissipation of energy by defects, impurities, and roughness of the surface of the resonator [9]. High temperature annealing and etching

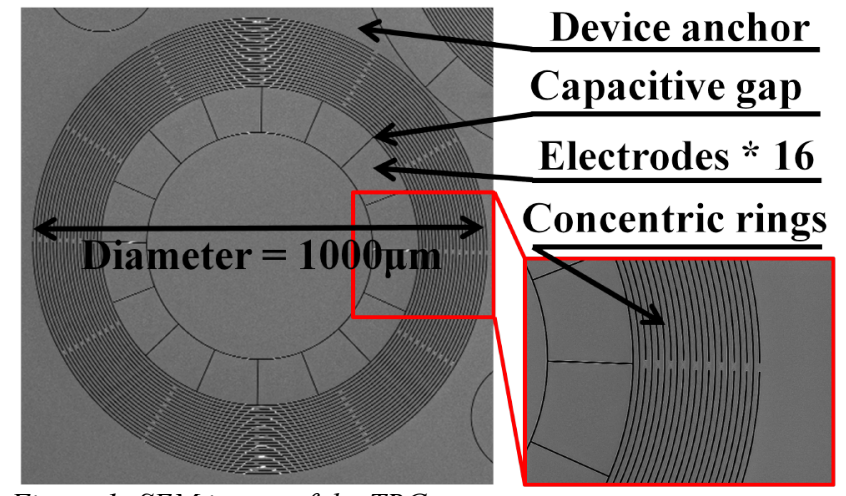

Figure 1: SEM image of the TRG.

of the device [10], as well as surface reflow [11] have been experimentally proven to reduce the surface loss of the resonators.

All the factors mentioned above contribute to the overall energy dissipation of resonators. In practice, it is difficult to experimentally identify the dominant dissipation mechanism [2]. As a result, although many techniques have been developed to reduce dissipative sources of resonators, they cannot be effectively applied without first identifying the dominant energy dissipation mechanism [12].

In this paper, we present a study on the energy dissipation mechanisms of the TRG. We utilize a previously demonstrated method which relies on measurements of quality factor as a function of temperature from the room temperature down to cryogenic temperatures, for encapsulated and vented devices, and separate measurements of the quality factor as a function of pressure near the room temperature [13].

\section{TOROIDAL RING GYROSCOPE}

The TRG was selected for its structural simplicity and a demonstrated potential for high performance [14]. It consists of a concentric ring structure, an outer anchor that encircles the rings, and an inner electrode assembly. The outer anchor structure leads to a more robust support of the structure compared to devices with a central anchor. The device was fabricated using the Epi-Seal process [15], which utilizes epitaxially grown silicon to seal the device layer at extremely high temperatures, resulting in an ultra-clean wafer-level sealing. The device was fabricated on single-crystal $<111>$ silicon wafers to achieve a better structural symmetry, enabling the operation in the $n=2$ mode. The SEM image of the device is shown in Fig 1. The diameter of the ring structure is $1000 \mu \mathrm{m}$ and it includes 20 concentric rings with a width of $6 \mu \mathrm{m}$. The rings are connected to each other with 6 spokes in between two consecutive rings. The thickness of the device is $60 \mu \mathrm{m}$ and the gaps in between the rings are $1.5 \mu \mathrm{m}$. The designed resonant frequency of the $\mathrm{n}=2$ mode of the TRG was $68.7 \mathrm{kHz}$ and the experimentally measured frequency was $54.5 \mathrm{kHz}$. The difference in frequency is possibly due to the over-etching of the device. 
One of the benefits of this specific design is that the $n=2$ mode is the mode with the lowest natural frequency. It comes before all the out-of-plane modes because of the large thickness-to-width ratio of the rings. It also comes before the $n=1$ rocking mode due to the phenomenon of mode ordering [16]. This order of the modes helps to improve the common mode rejection of external accelerations.

\section{ENERGY DISSIPATION ANALYSIS Viscous Air Damping}

Viscous air damping of a resonator is related to the pressure surrounding the resonator and its geometry. Although devices fabricated in the Epi-Seal process are sealed with pressure near $0.1 \mathrm{~Pa}[15]$, the narrow gap and a low frequency motion of the beams of the resonator will induce some viscous damping, especially if other dissipation mechanisms are suppressed.

It is impossible to directly measure the pressure inside the cavity of the Epi-Seal devices. Therefore, we utilized the overall quality factor of the resonator as an indicator of the pressure. The quality factor of the sealed device was first measured over a wide temperature range. The quality factor at room temperature was found to be 128,000. Then, we used the Focused Ion Beam (FIB) to form a small hole through the cap, exposing the interior of the resonator to the surrounding atmosphere, as shown in Fig. 2.

The device was placed into a vacuum chamber with adjustable air pressure and the quality factor of the device was measured as a function of pressure at room temperature. The relation between the air pressure and the quality factor of the device is shown in Fig. 3. The figure shows that the quality factor of the device reached 170,000 with air pressure below $0.001 \mathrm{~Pa}$, indicating that viscous air damping was overwhelmed by other dissipation mechanisms at pressures below $0.001 \mathrm{~Pa}$. The original quality factor of 128,000 for the sealed device corresponded to a pressure level of $0.04 \mathrm{~Pa}$, providing a good estimate for the pressure in the cavity of the device before venting. To suppress the effects of viscous air damping on the overall quality factor, we tested the vented device at pressures below $0.0001 \mathrm{~Pa}$ in the following sections.

\section{Thermoelastic Damping}

Thermoelastic damping is related to the exchange of energy between temperature and elastic deformation fields in resonating structures. The coupling term between the two fields is the CTE of the material. It has been shown that the TED of a cantilever beam is proportional to the square of CTE of the material, as shown in Equation (2), where $\rho$ is the density, $C_{p}$ is the heat capacity, $\alpha$ is CTE, $T$ is the temperature, and $E$ is Young's modulus. For the TRG devices that we studied, the general principle of TED through CTE-coupling between the temperature and elastic deformation fields still applies although they have a more complicated geometry. Silicon is a material whose CTE is strongly dependent on the temperature and it crosses zero at temperature of near 123K [17]. Because of these effects, the TED contribution to the overall quality factor as a function of temperature should have a strong and recognizable signature, making it possible to quantify TED in this resonator.

$$
Q_{T E D} \propto \frac{\rho C_{p}}{\alpha^{2} T E}
$$

The encapsulated resonator was first placed in a vacuum chamber and the chamber was cooled down by conductive contact with a liquid Nitrogen reservoir. The device was characterized at different ambient temperatures ranging from $80 \mathrm{~K}$ to $300 \mathrm{~K}$. The quality factor of the device was measured by the ring-down method for better accuracy while the temperature slowly rose due to the ambient temperature of the room. The device was tested before and

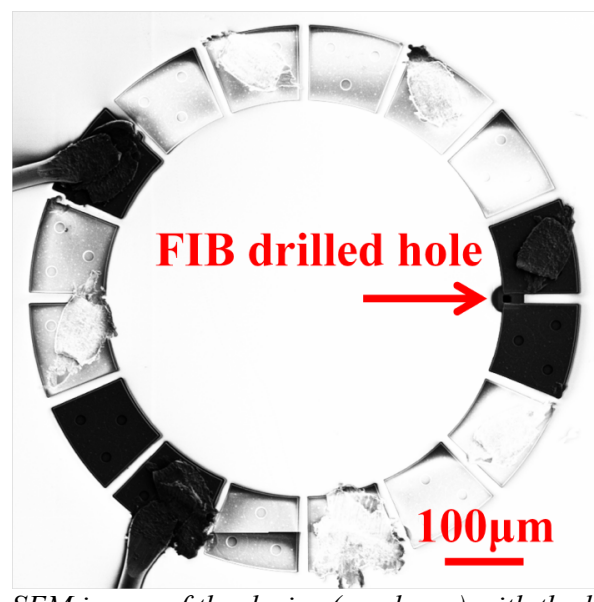

Figure 2: SEM image of the device (cap layer) with the hole drilled by FIB.

after the FIB-venting of the device. The relations between the temperature and the quality factor of the device, before and after venting, are shown in Fig. 4.

The device showed a higher quality factor after venting and operating below $0.0001 \mathrm{~Pa}$, as expected from the earlier pressure-sweep results in Fig. 3. Overall, the difference between the Q-T curves for the sealed and vented device was consistent with the expected temperature dependence of the viscous air damping, which should be inversely proportional to the square root of temperature. The magnitude of viscous air damping could be estimated by the difference of the two curves.

The maximum quality factor for the vented device rose to $1.35 \mathrm{M}$ at temperature of around $123 \mathrm{~K}$, and then decreased again at lower temperatures. This was the expected signature of a strong contribution from TED. The same signature was present in the data from the sealed device, but the additional contribution from viscous air damping suppressed the signature.

A model for TED in the TRG device was developed with Finite Element Analysis (FEA) in COMSOL MultiPhysics Package. The thermo-mechanical module was utilized to model the coupling of thermal and elastic deformation fields. At room temperature, the model predicted a Q of 200,000, which was in reasonable agreement with the experimental result of 170,000. Using the full set of temperature-dependent material parameters [17], prediction of the

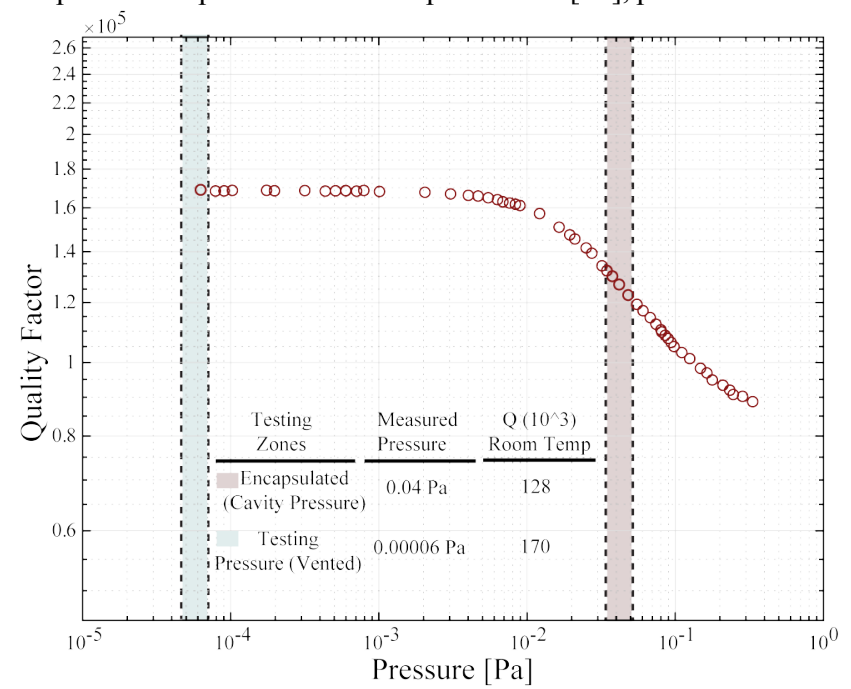

Figure 3: The relation between the air pressure and the quality factor of the device at room temperature. 


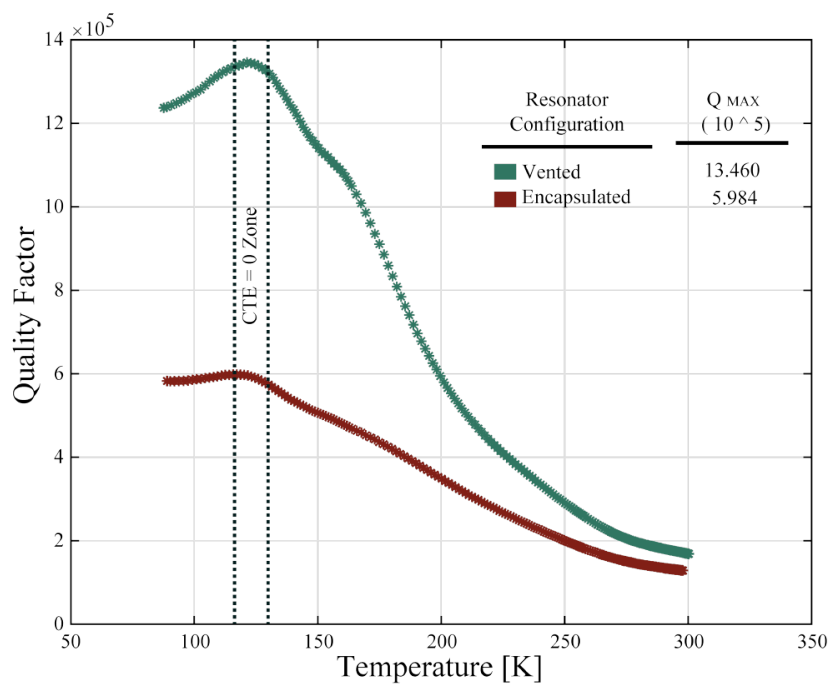

Figure 4: The relation between the temperature and the quality factor of the device before and after the device was vented by FIB.

temperature dependence of the TED contribution to the quality factor was built, and is plotted along with other models in Fig. 7.

At the peak of the Q-T curve for the vented device, we effectively suppressed contributions from viscous damping and TED, which left the anchor loss as the most likely remaining source of dissipation. This data alone was not sufficient to positively identify the anchor loss as the limiting mechanism at $\mathrm{Q}=1.35 \mathrm{M}$.

\section{Anchor loss}

All of the most commonly-discussed models for anchor loss depend only on material's mechanical properties, such as Young's modulus, and geometry. In this study, the relevant material properties and geometries were almost invariant over a large temperature range, so we hypothesized that the anchor loss was temperature-invariant.

To confirm this hypothesis, the resonant frequency of the device was measured over a large temperature range from $80 \mathrm{~K}$ to $300 \mathrm{~K}$. An overall frequency shift of only $64 \mathrm{~Hz}$ was demonstrated over a $220 \mathrm{~K}$ temperature range. The results are shown in Fig. 5. The very small shift in frequency $(<1300 \mathrm{ppm})$ indicated a weak temperature dependence of the mechanical properties of silicon, such as Young's modulus and Poisson's ratio.

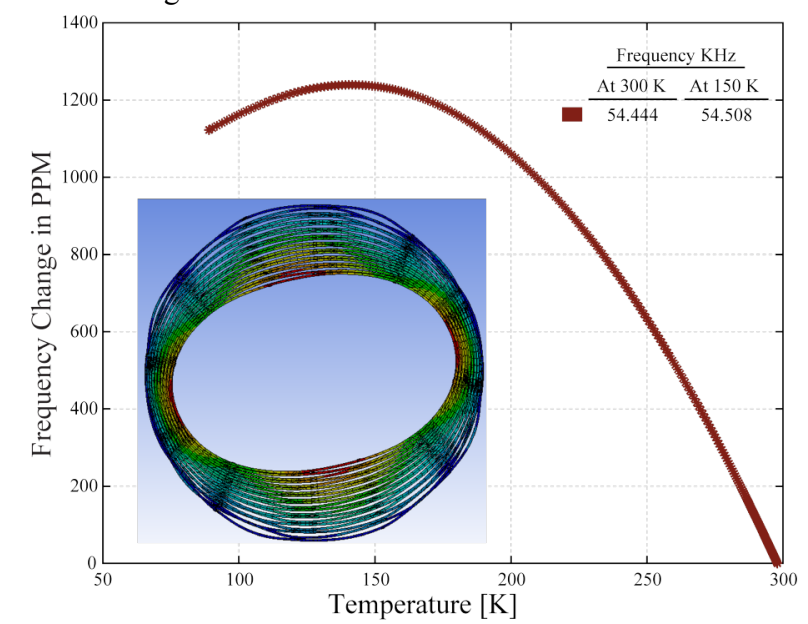

Figure 5: The relation between the temperature and the resonant frequency of the encapsulated device. Inserted is the mode shape of $n=2$ mode of TRG.

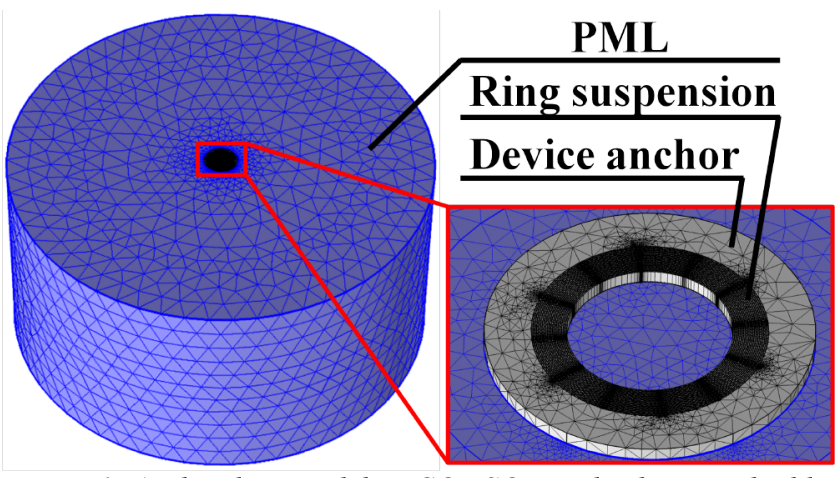

Figure 6: Anchor loss model in COMSOL MultiPhysics. The blue part is $P M L$.

In order to help to confirm that the dissipation mechanism limiting the quality factor to $1.35 \mathrm{M}$ is the anchor loss, we turned to the FEA model for anchor loss analysis in COMSOL MultiPhysics Package with the Perfectly Matched Layer (PML) model. In this model, the PML was built in a cylinder shape with the height of $15 \mathrm{~cm}$ and diameter of $30 \mathrm{~cm}$. Even though these dimensions far exceed the dimensions of the physical die, they were selected to be $20 \%$ greater than the wavelength in silicon at $68 \mathrm{kHz}(0.12 \mathrm{~m})$. The PML model is presented in Fig. 6.

In one set of simulations, the anchor loss model showed a quality factor limit of $1.32 \mathrm{M}$ with meshing errors of less than $5 \%$, which agreed with the highest quality factor of the device after venting and cooling down to $123 \mathrm{~K}$ (Fig. 4). Although the model matched the experimental results, there are still many free parameters in the model, such as the size of the substrate, the scaling factor and the shape of PML, that have not been explored. Based on this, we believe that the PML model in COMSOL Multiphysics does not refute our conclusion, but is not predictive enough to provide a strong confirmation.

\section{OVERALL QUALITY FACTOR ANALYSIS}

The overall quality factor of a resonator is related to many energy dissipation mechanisms. For the TRG device that we tested, the effects of Akhiezer damping and Landau-Runer damping were not considered since the $f^{*} \mathrm{Q}$ product is far too small. Surface loss could also be eliminated due to the ultra-clean cavity fabricated in the Epi-Seal process. Ohmic dissipation was also unlikely since the quality factor measurement was independent of the bias voltages used during testing. Therefore, our model for analysis of the quality factor only considered the effects of viscous air damping, TED, and the anchor loss.

The strongest test of the validity of the decision to neglect other dissipation mechanisms and to focus just on the viscous air damping, TED, and the anchor loss is a comparison between the $\mathrm{Q}(\mathrm{T})$ predicted by the model and the experimental results. We utilized the TED model described above with no adjustable parameters; to this we added a temperature-independent term representing the anchor loss, where the magnitude was taken from the peak of the experimental $\mathrm{Q}(\mathrm{T})$ curve, and was shown to be consistent with the PML model result. In Fig. 7, we compared the reciprocal sum of the TED and the constant anchor loss term with the experimentally measured $\mathrm{Q}(\mathrm{T})$ for the vented device (blue points, red curve), and close match with errors less than 5\% was demonstrated over the entire temperature range. This is a very strong confirmation that our experiment is well-represented by the reciprocal sum of TED and a temperature-independent anchor loss term, and that there are no other residual dissipation mechanisms playing a significant role. 


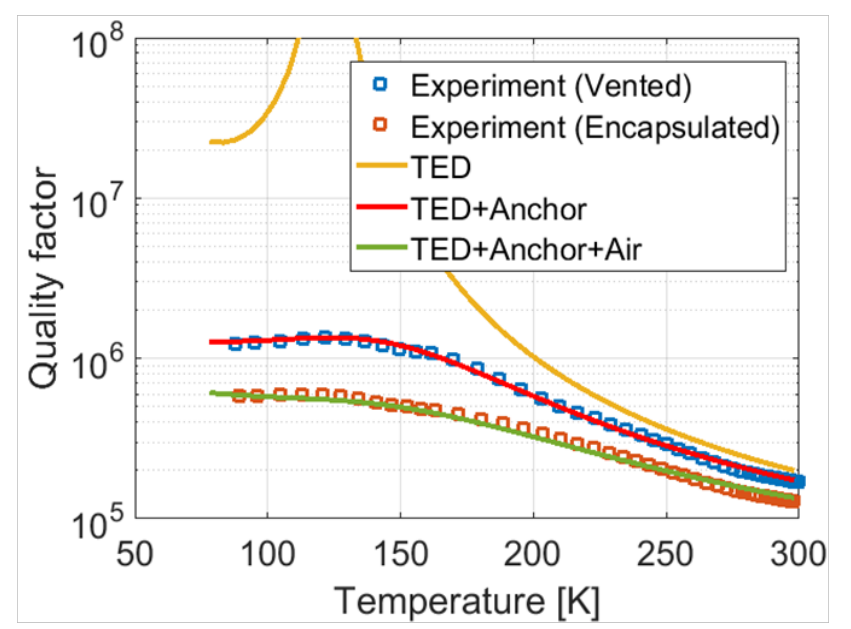

Figure 7: Comparison between the estimated $Q-T$ relation and the measured $Q-T$ relation.

Finally, we added a viscous air damping term to the reciprocal sum. The magnitude of the air damping was determined by the $\mathrm{Q}(\mathrm{P})$ measurement at room temperature, and we asserted a $1 /\left(\mathrm{T}^{1 / 2}\right)$ scaling. Again, the experimental result for the encapsulated device was an extremely good fit to this model with errors less than $10 \%$.

The agreement between model and experiment, including the qualitative shape and the quantitative match, is the strongest possible confirmation that the explanation for the results is valid.

\section{CONCLUSION}

This study follows a recently-established methodology for analysis of the energy dissipation mechanism in resonant MEMS devices [13], which includes measurements of the $\mathrm{Q}(\mathrm{T})$ over a wide temperature range for sealed and vented devices, and measurements of Q(P) for the vented device. In this study, we analyzed the energy dissipation mechanisms of a TRG device. The viscous air damping, TED, and the anchor loss were identified as the major loss mechanisms that affected the overall quality factor of the device. The value of each damping mechanism was estimated and a complete model representing the relation between the quality factor and temperature was constructed. Close match between the model and the experimental results confirmed the validity of the model.

\section{ACKNOWLEDGEMENT}

This work was supported by the Defense Advanced Research Projects Agency (DARPA) and U.S. Navy under Contract No. N66001-16-1-4021 at UC Irvine and Contract No. N66001-16-1-4023 at Stanford University. The fabrication work was performed in part at the Stanford Nanofabrication Facility (SNF), supported by the National Science Foundation under Grand ECS- 9731293. Special thanks to all the staff at SNF for their help during the fabrication process.

\section{REFERENCES}

[1] M. Weinberg, R. Candler, S. Chandorkar, J. Varsanik, T. Kenny, and A. Duwel. "Energy Loss in MEMS Resonators and the Impact on Inertial and RF Devices". Solid-State Sensors, Actuators and Microsystems Conference (TRANSDUCER), Denver, CO, 6/21-25/09, IEEE, (2009), pp. 688-695.

[2] J. Yang, T. Ono, and M. Esashi, "Energy dissipation in submicrometer thick single-crystal silicon cantilevers," IEEE Journal of Microelectromechanical systems, 11, 6, (2002).

[3] C. Zener, "Internal friction in solids. I. Theory of internal friction in reeds," Physical review, 52, 3, (1937).
[4] C. Zener, "Internal friction in solids II. General theory of thermoelastic internal friction," Physical Review, 53, 1, (1938).

[5] D. D. Gerrard, Y. Chen, S. A. Chandorkar, G. Yu, J. Rodriguez, I. B. Flader, D. D. Shin, C. D. Meinhart, O. Sigmund, and T. W. Kenn,. "Topology optimization for reduction of thermo-elastic dissipation in MEMS resonators," Solid-State Sensors, Actuators and Microsystems (TRANSDUCERS), Kaohsiung, Taiwan, 6/18-22/17, IEEE, (2017), pp. 794-797.

[6] D. Senkal, M. J. Ahamed, M. H. Asadian, S. Askari, and A. M. Shkel, "Demonstration of 1 Million Q-Factor on Microglassblown Wineglass Resonators With Out-of-Plane Electrostatic Transduction," IEEE Journal of Microelectromechanical Systems, 24, 1, (2015).

[7] S. A. Zotov, B. R. Simon, I. P. Prikhodko, A. A. Trusov, and A M. Shkel, "Quality Factor Maximization through Dynamic Balancing of Tuning Fork Resonator," IEEE Sensors Journal, 14, 8, (2014).

[8] A. Darvishian, B. Shiari, J. Y. Cho, T. Nagourney, and K. Najafi, "Anchor loss in hemispherical shell resonators". IEEE Journal of Microelectromechanical Systems, 26, 1, (2017).

[9] G. Palasantzas, "Quality factor due to roughness scattering of shear horizontal surface acoustic waves in nanoresonators," Journal of Applied Physics, 104, 5, (2008).

[10] J. J. Bernstein, M. G. Bancu, J. M. Bauer, E. H. Cook, P. Kumar, E. Newton, T. Nyinje, G. E. Perlin, J. A. Ricker, W.A. Teynor, and M. S. Weinberg, "High Q diamond hemispherical resonators: fabrication and energy loss mechanisms." Journal of Micromechanics and Microengineering, 25, 8, (2015).

[11] Y. Wang, M. H. Asadian, and A. M. Shkel, "Modeling the Effect of Imperfections in Glassblown Micro-Wineglass Fused Quartz Resonators," Journal of Vibration and Acoustics, 139, 4, (2017).

[12] Y. Wang, A. M. Shkel, "Study on Surface Roughness Improvement of Fused Quartz After Thermal and Chemical Post-Processing," IEEE Inertial Sensors, Laguna Beach, CA, 2/23-25/16, IEEE, (2016), pp. 101-104.

[13] J. Rodriguez, D. D. Gerrard, S. Chandorkar, Y. Chen, G. M. Glaze, I. B. Flader, C. H. Ahn, E. J. Ng, and T. W. Kenny, "Wide-range temperature dependence studies for devices limited by thermoelastic dissipation and anchor damping," Solid-State Sensors, Actuators and Microsystems (TRANSDUCERS), Kaosiung, Taiwan, 6/18-22/17, IEEE, (2017), pp. 1100-1103.

[14] D. Senkal, S. Askari, M. J. Ahamed, E. Ng, V. Hong, Y. Yang, C. H. Ahn, T. W. Kenny, and A. M. Shkel, "100k Q-Factor Toroidal Ring Gyroscope Implemented in Wafer-level Epitaxial Silicon Encapsulation Process, " IEEE MEMS Conference, San Francisco, CA, 1/26-30/14, IEEE, (2014), pp. 24-27.

[15] Y. Yang, E. J. Ng, Y. Chen, I. B. Flader, and T. W. Kenny "A Unified Epi-seal Process for Fabrication of High-stability Microelectromechanical Devices," IEEE Journal of Microelectromechanical Systems, 25, 3, (2016).

[16] A. Efimovskaya, D. Wang, Y. Lin, and A. M. Shkel, "On Ordering of Fundamental Wineglass Modes in Toroidal Ring Gyroscope," IEEE Sensors Conference, Orlando, FL, 10/30-11/2/16, IEEE, (2016), pp. 1-3.

[17] T. Middelmann, A. Walkov, G. Bartl, and R. Schödel, "Thermal expansion coefficient of single-crystal silicon from 7 K to 293 K," Physical Review B, 92, 17, (2015).

\section{CONTACT}

*Yusheng Wang, tel: +1-949-562-9560; yushengw@uci.edu 